\title{
Global asymptotic stability of the fractional differential equations
}

\section{Ndolane Sene}

Laboratoire Lmdan, Département de Mathématiques de la Décision, Université Cheikh Anta Diop de Dakar, BP 5683 Dakar Fann, Senegal.

\begin{abstract}
In this note, we present a global asymptotic stability criterion for the fractional differential equations in triangular form. We use the Caputo generalized fractional derivative in our investigations. In our note, we introduce a new procedure to study the global asymptotic stability of the fractional differential equations.
\end{abstract}

Keywords: Caputo left generalized fractional derivative, fractional differential equations with input, Mittag-Leffler input stability.

2010 MSC: 93D25, 93D05, 26A33.

(C)2020 All rights reserved.

\section{Introduction}

The fractional differential equations attract many mathematicians due to its novelty. Many problems related to the differential equations described by a specified derivative can be studied. The first problem consists of proving the existence and the uniqueness of the solution of the fractional differential equations [4]. The second problem is to solve the fractional differential equations. Many methods exist to solve the fractional differential equations. We have homotopy perturbation method [16], Laplace transform method [13], Fourier transformation method [14], Fourier sine transformation method [14], and many others. An important problem related to the solution fractional differential equations is to analyze the behavior of the analytical solutions. The issue is to study the stability and convergence. The stability analysis of the fractional differential equations has received many investigations in literature $[8,9,11,13,18]$. For recent investigations in stability analysis, see the following papers [3,18]. The contribution of this note is to propose a new procedure to analyze the global asymptotic stability of the fractional differential equations described by the Caputo generalized fractional differential equations. The use of the classical condition $|\arg (\lambda(A))|>\frac{\alpha \pi}{2}[13]$ is not trivial when the given fractional differential equation is in high dimension or admit a nonlinear term. The issue is to rewrite the given fractional differential equation in its triangular form (if possible). After decomposition, for each sub fractional differential equation, we study its stability.

Email address: ndolanesene@yahoo.fr (Ndolane Sene)

doi: $10.22436 /$ jnsa.013.03.06

Received: 2019-11-05 Revised: 2019-11-19 Accepted: 2019-11-27 
This note proposes a new method to provide the stability of the initial fractional differential equation. In this note, we consider a fractional differential equation. We rewrite it in two-dimensional space. We suppose the first sub fractional differential equation is fractional input stable [10,12,13]. And the second sub fractional differential equation is global asymptotic stable [13]. We try to prove when we combine fractional input stability and the global asymptotic stability; then the initial fractional differential equation is globally asymptotically stable.

The note is structured as follows. In Section 2, we introduce the result of this note. In Section 3, we illustrate our result by an example. In Section 4, we give an open problem for future works.

\section{Result and discussion}

This note is to give useful and helpful remarks on the global asymptotic stability of the fractional differential equation in triangular form. Let's recall some definitions and lemmas which will be used to prove our result. Consider the differential equation described by the Caputo-Liouville generalized fractional derivative defined by

$$
\left\{\begin{array}{l}
D_{c}^{\alpha, \rho} x_{1}=f\left(x_{1}, x_{2}\right) \\
D_{c}^{\alpha, \rho} x_{2}=f_{2}\left(x_{2}\right)
\end{array}\right.
$$

where $x_{i} \in \mathbb{R}$ with $1 \leqslant i \leqslant 2$. The $D_{\mathcal{c}}^{\alpha, \rho}$ denotes the Caputo-Liouville generalized fractional derivative $[2,5-7]$ represented by

$$
\left(D_{c}^{\alpha, \rho} f\right)(t)=\frac{1}{\Gamma(1-\alpha)} \int_{0}^{t}\left(\frac{t^{\rho}-s^{\rho}}{\rho}\right)^{-\alpha} f^{\prime}(s) d s,
$$

for all $t>0$, where the order $\alpha \in(0,1)$ and $\Gamma(\cdots)$ is the gamma function. Let's recall some fundamental definitions and the comparison functions.

Definition 2.1 ([8]). The Mittag-Leffler function with two parameters is defined by the series

$$
\mathrm{E}_{\alpha, \beta}(z)=\sum_{\mathrm{k}=0}^{\infty} \frac{z^{\mathrm{k}}}{\Gamma(\alpha \mathrm{k}+\beta)},
$$

where the parameters $\alpha>0, \beta \in \mathbb{R}$ and $z \in \mathbb{C}$. We recover the classical exponential function by letting $\alpha=\beta=1$.

Definition $2.2([1,13,15,17])$. The class $\mathcal{P D}$ function denotes the set of all continuous functions $\alpha: \mathbb{R}_{\geqslant 0} \rightarrow$ $\mathbb{R}_{\geqslant 0}$ satisfying $\alpha(0)=0$, and $\alpha(s)>0$ for all $s>0$. A class $\mathcal{K}$ function is an increasing $\mathcal{P D}$ function. The class $\mathcal{K}_{\infty}$ represents the set of all unbounded $\mathcal{K}$ functions.

Definition 2.3 ([1, 13, 15, 17]). A continuous function $\beta: \mathbb{R}_{\geqslant 0} \times \mathbb{R}_{\geqslant 0} \rightarrow \mathbb{R}_{\geqslant 0}$ is said to be of class $\mathcal{K} \mathcal{L}$ if $\beta(., t) \in \mathcal{K}$ for any $t \geqslant 0$, and $\beta(s,$.$) is non-increasing and tends to zero as its arguments tend to infinity.$

Let's recall the definitions of the stability notions which will be used in this section to prove our main result.

Definition 2.4 ([13]). The fractional differential equation defined by Eq. (2.1) is said to be generalized globally asymptotically stable if there exists a class $\mathcal{K} \mathcal{L}$ function $\beta$ such that for any initial condition $\xi$, the following inequality holds

$$
\|x(t, \xi)\| \leqslant \beta\left(\|\xi\|, t^{\rho}-t_{0}^{\rho}\right),
$$

where $\|$.$\| represents the Euclidean norm.$

Definition 2.5 ([11, 13]). The fractional differential equation defined by Eq. (2.1) is said to be generalized fractional input stable if, for any input $u \in \mathbb{R}^{\mathrm{m}}$, there exist a class $\mathcal{K} \mathcal{L}$ function $\beta$ and a $\mathcal{K}_{\infty}$ function $\gamma$, such that for any initial condition $\xi$, its solution satisfies

$$
\|x(t, \xi, u)\| \leqslant \beta\left(\|\xi\|, t^{\rho}-t_{0}^{\rho}\right)+\gamma(\|u\|) .
$$


We introduce the main result of this note. We found many properties related to the generalized fractional input stability and generalized asymptotic stability in [11, 13].

Theorem 2.6. Consider the fractional differential equation defined by $D_{c}^{\alpha, \rho} x_{1}=f_{1}\left(x_{1}, x_{2}\right)$ is fractional input stable respecting the input $\left\|\mathrm{x}_{2}\right\|$ and the fractional differential equation $\mathrm{D}_{\mathrm{c}}^{\alpha, \rho} \mathrm{x}_{2}=\mathrm{f}_{2}\left(\mathrm{x}_{2}\right)$ is globally asymptotically stable. Then the fractional differential equation defined by Eq. (2.1) is globally asymptotically stable.

Proof. From the global asymptotic stability of the fractional differential equation defined by $D_{c}^{\alpha, \rho} x_{1}=$ $f_{2}\left(x_{2}\right)$, we have $\lim _{t \rightarrow+\infty}\left\|x_{2}(t)\right\|=0$. From the fractional input stability of the fractional differential equation defined by $D_{\mathcal{c}}^{\alpha, \rho} x_{1}=f_{1}\left(x_{1}, x_{2}\right)$, we obtain the property converging-input converging state [12, 13]. From which it follows that $\lim _{t \rightarrow+\infty}\left\|x_{1}(t)\right\|=0$. Finally, the solution of the fractional differential equation (2.1) is convergent. That is $\lim _{t \rightarrow+\infty}\|x(t)\|=0$.

Secondly, from global asymptotic stability of the fractional differential equation defined by $D_{\mathcal{c}}^{\alpha, \rho} x_{1}=$ $f_{2}\left(x_{2}\right)$, there exists a class $\mathcal{K} \mathcal{L}$ function $\beta_{2}$ such that for any initial condition $\xi_{2}$, the following inequality holds

$$
\left\|x_{2}(t)\right\| \leqslant \beta_{2}\left(\left\|\xi_{2}\right\|, t^{\rho}\right) \leqslant \beta_{2}\left(\left\|\xi_{2}\right\|, 0\right) .
$$

From the fractional input stability of the fractional differential equation defined by $D_{c}^{\alpha, \rho} x_{1}=f_{1}\left(x_{1}, x_{2}\right)$, there exist a class $\mathcal{K} \mathcal{L}$ function $\beta_{1}$ and a $\mathcal{K}_{\infty}$ function $\gamma$, such that for any initial condition $\xi_{1}$, its solution satisfies

$$
\left\|x_{1}(t)\right\| \leqslant \beta_{1}\left(\left\|\xi_{1}\right\|, t^{\rho}\right)+\gamma\left(\left\|x_{2}\right\|_{\infty}\right) \leqslant \beta_{1}\left(\left\|\xi_{1}\right\|, 0\right)+. \gamma\left(\left\|\beta_{2}(\|\xi\|, 0)\right\|\right) .
$$

From Eqs. (2.2) and (2.3), we have proved there exists $\epsilon$ such that $\|x(t)\| \leqslant \epsilon$, thus the fractional differential equation (2.1) is stable. Combining the stability and the convergence, we conclude the fractional differential equation (2.1) is globally asymptotically stable.

\section{Illustration and discussion}

Consider the fractional differential equation described by the Caputo generalized fractional derivative defined by

$$
\left\{\begin{array}{l}
\mathrm{D}_{\mathrm{c}}^{\alpha, \rho} \mathrm{x}_{1}=-\mathrm{x}_{1}+\mathrm{x}_{2} \\
\mathrm{D}_{\mathrm{c}}^{\alpha, \rho} \mathrm{x}_{2}=-2 \mathrm{x}_{2}
\end{array}\right.
$$

where $x_{1}, x_{2} \in \mathbb{R}$. The matrix associated with the fractional differential equation (3.1) is defined by

$$
A=\left(\begin{array}{cc}
-1 & 1 \\
0 & -2
\end{array}\right)
$$

The matrix $A$ satisfies the condition $|\arg (\lambda(A))|>\frac{\alpha \pi}{2}$. Thus, the fractional differential equation defined by Eq. (3.1) is globally asymptotically stable. The utility of this note is, in many cases, we have noted when the dimension of the fractional differential equation becomes too high $n \geqslant 3$, analyzing the global asymptotic stability becomes not trivial. The issue is to rewrite the fractional differential equation in its triangular form. We study the stability of each sub fractional differential equations and use our Theorem.

Let's apply our Theorem. Firstly, the linear fractional differential equation $D_{c}^{\alpha, \rho} x_{2}=-2 x_{2}$ is clearly global asymptotic stability. Secondly, the fractional differential equation $D_{c}^{\alpha, \rho} x_{1}=-x_{1}+x_{2}$ is fractional input stable when we consider $\left\|x_{2}\right\|$ as an exogenous input. To see the fractional input stability, we determine the solution of the fractional differential equation $D_{c}^{\alpha, \rho} x_{1}=-x_{1}+x_{2}$. The solution of the fractional differential equation $D_{c}^{\alpha, \rho} x_{1}=-x_{1}+x_{2}$ is given by the following expression [13]

$$
x_{1}(t)=\xi E_{\alpha}\left(A\left(\frac{t^{\rho}}{\rho}\right)^{\alpha}\right)+\int_{0}^{t}\left(\frac{t^{\rho}-s^{\rho}}{\rho}\right)^{\alpha-1} E_{\alpha, \alpha}\left(A\left(\frac{t^{\rho}}{\rho}\right)^{\alpha}\right) x_{2}(s) \frac{d s}{s^{1-\rho}} .
$$

Applying the norm, see the reasoning in $[12,13]$, we arrives to

$$
\left\|x_{1}(t)\right\| \leqslant \beta_{1}\left(\left\|\xi_{1}\right\|, t^{\rho}\right)+M\left\|x_{2}\right\|,
$$


where $\beta_{1}\left(\left\|\xi_{1}\right\|, t^{\rho}\right)=\xi E_{\alpha}\left(A\left(\frac{t^{\rho}}{\rho}\right)^{\alpha}\right)$ and $M$ is constant number obtained after calculation done on Eq. (3.2). Clearly, we conclude the fractional differential equation $D_{c}^{\alpha, \rho} x_{1}=-x_{1}+x_{2}$ is fractional input stable. From our Theorem 2.6, we conclude the fractional differential equation (3.1) is globally asymptotically stable.

For another example when the Theorem 2.6 is useful, consider the fractional differential equation with nonlinear term described by the Caputo generalized fractional derivative defined by

$$
\left\{\begin{array}{l}
D_{c}^{\alpha, \rho} x_{1}=-x_{1}^{3}-x_{1}+2 x_{2} \\
D_{c}^{\alpha, \rho} x_{2}=-x_{2}
\end{array}\right.
$$

where $x_{1}, x_{2} \in \mathbb{R}$. Using the Lyapunov characterization method, one can prove the fractional differential equation is globally asymptotically stable, but the proof is not trivial. Theorem 2.6 is easier to be applied. We know $D_{c}^{\alpha, \rho} \chi_{2}=-\chi_{2}$ is global asymptotic stable, we can use the Lyapunov function defined by $V\left(x_{2}\right)=$ $\frac{x_{2}^{2}}{2}[13]$. To see it, the derivative of the function $V$ along the trajectories yields that

$$
\mathrm{D}_{\mathrm{c}}^{\alpha, \rho} \mathrm{V}(\mathrm{x}) \leqslant-\chi_{2}^{2}<0
$$

From which the Lyapunov characterization gives the trivial solution of the fractional differential equation $\mathrm{D}_{\mathrm{c}}^{\alpha, \rho} \chi_{2}=-\chi_{2}$ is globally asymptotically stable. The second fractional differential equation is fractional input stable, we can see it by using again the Lyapunov function $V\left(x_{1}\right)=\frac{x_{1}^{2}}{2}[11,12]$. We have

$$
\mathrm{D}_{\mathrm{c}}^{\alpha, \rho} \mathrm{V}(\mathrm{x}) \leqslant-x_{1}^{4}-x_{1}^{2}+2 x_{1} x_{2} \leqslant-x_{1}^{4}-x_{1}^{2}+x_{1}^{2}+x_{2}^{2} \leqslant-x_{1}^{4}+x_{2}^{2} \leqslant-(1-\theta) x_{1}^{4}-\theta x_{1}^{4}+x_{2}^{2}
$$

with $\theta \in(0,1)$. We observe

$$
\left\|x_{1}\right\| \geqslant\left[\frac{\left\|x_{2}\right\|}{\theta}\right]^{1 / 2} \Longrightarrow D_{c}^{\alpha, \rho} V(x) \leqslant-(1-\theta) x_{1}^{4}
$$

From which we get the fractional input stability of the fractional differential equation defined by $D_{c}^{\alpha, \rho} x_{1}=$ $-x_{1}^{3}-x_{1}+2 x_{2}$. Using Theorem 2.6, we conclude that the fractional differential equation (3.3) is GAS.

\section{Open problem}

We consider the fractional differential equation in two-dimensional spaces in Theorem 2.6. The future direction of this work is to extend Theorem 2.6 to high dimensional spaces $n \geqslant 3$.

\section{References}

[1] T. Abdeljawad, F. Madjidi, F. Jarad, N. Sene, On Dynamic Systems in the Frame of Singular Function Dependent Kernel Fractional Derivatives, Mathematics, 7 (2019), 13 pages. 2.2, 2.3

[2] Y. Adjabi, F. Jarad, T. Abdeljawad, On generalized fractional operators and a Gronwall type inequality with applications, Filomat, 31 (2017), 5457-5473. 2

[3] A. Ben Makhlouf, Stability with respect to part of the variables of nonlinear Caputo fractional differential equations, Math. Commun., 23 (2018), 119-126. 1

[4] Y. Y. Gambo, R. Ameen, F. Jarad, T. Abdeljawad, Existence and uniqueness of solutions to fractional differential equations in the frame of generalized Caputo fractional derivatives, Adv. Difference Equ., 2018 (2018), 13 pages. 1

[5] F. Jarad, T. Abdeljawad, A modified laplace transform for certain generalized fractional operators, Results Nonlinear Anal., 2 (2018), 88-98. 2

[6] F. Jarad, E. Uğurlu, T. Abdeljawad, D. Baleanu, On a new class of fractional operators, Adv. Difference Equ., 1 (2017), 16 pages.

[7] U. N. Katugampola, New approach to a generalized fractional integral, Appl. Math. Comput., 218 (2011), 860-865. 2

[8] N. Sene, Exponential form for lyapunov function and stability analysis of the fractional differential equations, J. Math. Computer Sci., 18 (2018), 388-397. 1, 2.1 
[9] N. Sene, Lyapunov characterization of the fractional nonlinear systems with exogenous input, Fractal Fract., 2 (2018), 10 pages. 1

[10] N. Sene, Fractional input stability for electrical circuits described by the Riemann-Liouville and the Caputo fractional derivatives, AIMS Math., 4 (2019), 147-165. 1

[11] N. Sene, Fractional input stability and its application to neural network, Discrete Contin. Dyn. Syst. Ser. S, 13 (2019), 853-865. 1, 2.5, 2, 3

[12] N. Sene, Mittag-Leffler input stability of fractional differential equations and its applications, Discrete Contin. Dyn. Syst. Ser. S, 13 (2019), 867-880. 1, 2, 3, 3

[13] N. Sene, Stability analysis of the generalized fractional differential equations with and without exogenous inputs, J. Nonlinear Sci. Appl., 12 (2019), 562-572. 1, 2.2, 2.3, 2.4, 2.5, 2, 2, 3, 3, 3

[14] N. Sene, Solution of fractional diffusion equations and Cattaneo-Hristov diffusion model, Int. J. Anal. Appl., 17 (2019), 191-207. 1

[15] N. Sene, A. Chaillet, M. Balde, Relaxed conditions for the stability of switched nonlinear triangular systems under arbitrary switching, Systems Control Lett., 84 (2015), 52-56. 2.2, 2.3

[16] N. Sene, A. N. Fall, Homotopy Perturbation p-Laplace Transform Method and Its Application to the Fractional Diffusion Equation and the Fractional Diffusion-Reaction Equation, Fract. Frac., 3 (2019), 15 pages. 1

[17] N. Sene, G. Srivastava, Generalized Mittag-Leffler Input Stability of the Fractional Differential Equations, Symmetry, 11 (2019), 12 pages. $2.2,2.3$

[18] A. Souahi, A. Ben Makhlouf, M. A. Hammami, Stability analysis of conformable fractional-order nonlinear systems, Indag. Math. (N.S.), 28 (2017), 1265-1274. 1 